\title{
Sal e Hipertensión Arterial
}

\author{
Gloria Valdés Stromilli \\ Departamento de Nefrología, Escuela de Medicina, Pontificia Universidad Católica. \\ Recibido el 5 de marzo de 2009. Aceptado el 19 de marzo de 2009
}

Rev Chil Cardiol 2009; 28: 107-114

Llama la atención que a pesar de la experiencia clínica, y de numerosos estudios epidemiológicos, clínicos y de biología molecular que apoyan la asociación directa entre sodio $(\mathrm{Na})$ y presión arterial, aun persistan discrepancias sobre la efectividad e inocuidad de la restricción de sal para prevenir y tratar la hipertensión ${ }^{1}$. Es por esta razón que se justifica analizar críticamente esta relación y las recomendaciones que derivan de ella.

\section{Evidencias epidemiológicas}

Alrededor de 40 tribus primitivas que consumen menos de $3 \mathrm{~g}$ de sal o $\mathrm{NaCl} /$ día (de ahora en adelante expresada en $\mathrm{g}$ sal/día ${ }^{1}$ ) presentan estabilidad de la presión arterial a lo largo de la vida, y ausencia de hipertensión. En cambio, poblaciones japonesas con una ingesta promedio de $27 \mathrm{~g}$ tienen alta prevalencia del mejor marcador de riesgo hipertensivo, los accidentes cerebrales hemorrágicos. Con ingestas intermedias la prevalencia de hipertensión tiende a correlacionar con la ingesta de sal.

El mayor estudio dirigido a evaluar la asociación entre ingesta de sal y presión arterial, el INTERSALT2, 3 , incluyó a más de 10.000 sujetos, en 52 centros, en diferentes partes del mundo; en él la excreción urinaria se correlacionó con presión arterial entre individuos, pero no entre los distintos centros de estudio. La mayor excreción de Na se asoció al aumento de presión entre los 25 y los 55 años, demostrándose que una elevación de $6 \mathrm{~g}$ se acompaña de una elevación de las presiones sistólicas y diastólicas de 10 y $6 \mathrm{~mm} \mathrm{Hg}$, respectivamente.

El estudio CARDIAC (Cardiovascular Diseases and Alimentary Comparison), que incluyó a más de 7.000 individuos, mostró que la correlación entre ingesta de $\mathrm{Na}$ y presión arterial es significativa para sistólica y diastólica en hombres ${ }^{4}$, mientras que en mujeres esta correlación aparece después de la menopausia 5 , cuando la incidencia de hipertensión en mujeres iguala o supera la de los hombres.

Las poblaciones que migran desde sitios con baja ingesta de sal hacia lugares más desarrollados y con mayor consumo de sodio, han aumentado la presión en meses (ej. 7/6 mm Hg en Kenia6). Campesinos chinos que migraron a un área urbana, $\mathrm{y}$ aumentaron la ingesta en $6 \mathrm{~g}$ elevando sus presiones en $2.3 / 1.8 \mathrm{~mm} \mathrm{Hg}$.

A la inversa, el efecto benéfico de reducir la sal de la dieta en poblaciones ha sido demostrado en dos comunidades portuguesas, una de las cuales disminuyó la ingesta de 21 a $12 \mathrm{~g}$. Al cabo de 2 años

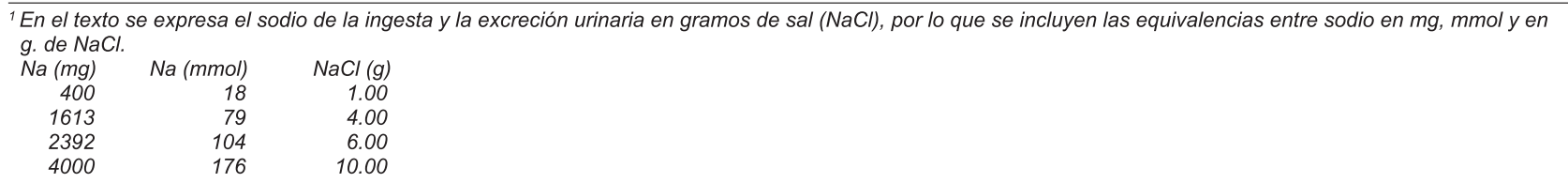

Correspondencia: Dra. Gloria Valdés Stromilli

Depto. de Nefrología

Escuela de Medicina, PUC

Correo Electrónico:gvaldes@med.puc.cl 
la comunidad intervenida presentó una reducción de $13 / 6 \mathrm{~mm} \mathrm{Hg}$ respecto a la comunidad control8.

Se postula que los negros que llegaron a Centro y Norteamérica fueron seleccionados de acuerdo a su capacidad de retener sal por las desastrosas condiciones sanitarias de los barcos negreros y de la vida en esclavitud. Esta característica que les permitió sobrevivir es la que haría que la hipertensión arterial sea más prevalente en la población afro-americana, y que ésta sea más respondedora a manejo de volumen que a intervención sobre el eje renina-angiotensina ${ }^{9}$.

\section{Estudios clínicos}

Kempner en 1948 usó una dieta de menos de $0.5 \mathrm{~g}$ de sal en 500 hipertensos, y obtuvo un $20 \%$ de descenso de las cifras tensionales en $62 \%$ de ellos, junto a disminución del diámetro cardíaco, y regresión de la retinopatía severa ${ }^{10}$. Hasta la aparición de los diuréticos en la década de los 50 , esta fue la primera intervención eficaz para controlar la hipertensión.

El estudio que mejor cuantificó la reducción de presión con una ingesta moderada de sal por 4 semanas, fue el realizado por MacGregor y colaboradores en 19 hipertensos con presiones basales de 156/98 mm Hg${ }^{9}$. A lo largo del estudio se mantuvo una excreción urinaria de $5 \mathrm{~g}$ de sal/24 horas, a la que se adicionó en forma ciega, randomizada, cruzada y controlada, con placebo por 4 semanas, un aporte oral de $5 \mathrm{~g}$ de sal. Las presiones bajo restricción fueron $7.1 \mathrm{~mm} \mathrm{Hg}$ (6.1\%), menores que al recibir el aporte de $\mathrm{Na}$. Un meta-análisis del efecto de una reducción moderada de sal (aproximadamente de $4 \mathrm{~g}$ ) que sólo incluyó estudios randomizados, observó un descenso de 5.0/3.0 $\pm 0.4 / 0.2$ en hipertensos $y$ 2.0/1.0 $\pm 0.30 / 0.2 \mathrm{~mm} \mathrm{Hg}$ en normotensos; en este meta-análisis se observó correlación entre el cambio de la ingesta de $\mathrm{Na}$ y el descenso de presión arterial, de modo que una reducción de $6 \mathrm{~g}$ predijo un descenso de $7.1 / 3.9 \mathrm{~mm} \mathrm{Hg}$ en hipertensos y $3.6 / 1.7 \mathrm{~mm} \mathrm{Hg}$ en normotensos ${ }^{11}$.

El mayor estudio que evaluó el efecto de niveles bajos, intermedios y elevados de $\mathrm{Na}(3,6$ y $9 \mathrm{~g})$, administrados por 30 días cada uno en forma randomizada, asociado o no a dieta DASH (Dietary Approach to Stop Hypertension), fue realizado en 412 adultos con presiones sistólicas entre 120 y 160 y diastólicas entre 80 y $95 \mathrm{~mm} \mathrm{Hg}$. Reducir el aporte de $\mathrm{Na}$ de 6 a $3 \mathrm{~g}$. causó un descenso de presión arterial sistólica mayor que el obtenido cuando se redujo de 9 a $6 \mathrm{~g}$ (-4.6 versus $-2.1 \mathrm{~mm} \mathrm{Hg}$ ); para presión diastólica el descenso fue mayor al descender de 9 a 6 , que de 6 a $3 \mathrm{~g}(-1.1 \text { versus } 0.6 \mathrm{~mm} \mathrm{Hg})^{12}$. La dieta DASH mostró un descenso tensional adicional, pero no sumatorio, de -7, -5 y -3.0 mm Hg para presión sistólica, y de -3.0, -2.0 y $-1.0 \mathrm{~mm} \mathrm{Hg}$ para diastólica para la ingesta de 9,6 y $3 \mathrm{~g}$, los que se pueden atribuir a otros cambios de esta dieta, como mayor consumo de potasio y a la reducción de grasas saturadas.

En general los efectos de reducir la sal son mayores en negros, individuos de edad media o mayores, obesos, diabéticos o insuficientes renales.

\section{Fisiopatología}

Los trabajos pioneros de Guyton ${ }^{13-15}$ en animales, y en modelos matemático-computacionales, describieron las relaciones simultáneas entre los múltiples sistemas de control circulatorio, y le permitieron postular categóricamente que no existe elevación permanente de la presión arterial a menos que se comprometa la excreción renal de Na por a) acción deletérea directa sobre el riñón, b) constricción de las arterias que perfunden el riñón, c) sobrecarga de Na y agua, d) supresión hormonal de la excreción de $\mathrm{Na}$, o por e) cambio electrolítico que influencie la función renal. El desplazamiento a la derecha de la curva presión arterial-natriuresis en hipertensos constituye una imagen clásica de la fisiopatología, y un paradigma que se puede aplicar a las distintas formas genéticas que se asocian a mayor reabsorción de $\mathrm{Na}$ en los distintos segmentos del riñón y a otras intervenciones (ej, acción de hipotensores, como bloqueadores del sistema renina-angiotensina, antagonistas de calcio, diuréticos, y medidas no farmacológicas como dieta DASH y suplementación de potasio).

La necesidad de emplear diuréticos en un gran número de pacientes para lograr un control óptimo de las cifras tensionales apoya el rol preponderante 
de una mayor reabsorción renal de sodio sobre el control de la presión arterial. Un modelo clínico que avala el rol central del riñón en la génesis de hipertensión es el del receptor de riñón de un donante con antecedente familiar de hipertensión, que tiene más posibilidades de desarrollar hipertensión que aquel que recibe el órgano de un donante sin historia familiar de hipertensión ${ }^{16}$.

El efecto de retención de sal generaría hipertensión por expansión de volumen, pero también por aumento de la reactividad arteriolar. En 1956, Tobian describió que las aortas de ratas hipertensas por nefrectomía unilateral y ligadura en 8 del riñón remanente presentaban mayor contenido de agua, $\mathrm{Na}, \mathrm{K}, \mathrm{Mg}$ y $\mathrm{P}$ que la de animales sometidos a la misma intervención que no presentaban hipertensión. Acuñó el concepto de "water logging", que al aumentar el grosor de la pared arterial elevaría la resistencia17. En 1977 Blaustein atribuyó este aumento de reactividad a mayor concentración intracelular de $\mathrm{Na}$, que se acompañaría de mayor $\mathrm{Ca}$ intracelular y mayor activación del aparato contráctil del músculo liso arterial18,19. Esta relación entre $\mathrm{Na}$ y Ca intracelular explicaría el efecto similar de diuréticos y bloqueadores de canales de $\mathrm{Ca}$.

Si bien los estudios epidemiológicos y clínicos demuestran la asociación entre ingesta de sal e hipertensión arterial, llama la atención que aún en las poblaciones que consumen las más altas ingestas de sal, existen porcentajes importantes de individuos que no desarrollan hipertensión, que pertenecen al grupo de resistentes a la sal. Esto demuestra que la alta ingesta de sal no es la causa, sino el gatillo de la hipertensión actuando sobre las características individuales.

¿Cuál es(son) la(s) causa(s) de la sensibilidad y de la resistencia a la sal?. Para contestar esta pregunta es necesario definir primero lo que se considera sensibilidad (SS) o resistencia a la sal (SR). De las diversas definiciones he seleccionado la de Luft y Weinberger 20 , quienes expandieron a normotensos e hipertensos con 2 litros de suero fisiológico intravenosos en 4 horas durante la mañana del primer día, midiendo presión arterial al término de la infusión, y luego depletaron con dieta de $0.5 \mathrm{~g}$ sal y 3 dosis de furosemida $40 \mathrm{mg}$ oral, para medir la presión al $3^{\text {er }}$ día. Las respuestas mostraron una distribución gaussiana, y se definió arbitrariamente sensibilidad a la sal al decremento de presión arterial media de 10 o más $\mathrm{mm} \mathrm{Hg}$, resistencia a la reducción menor o igual a $5 \mathrm{~mm} \mathrm{Hg}$, y respuesta indeterminada a los cambios intermedios. En normotensos se encontraron $26 \%$ SS y $58 \%$ SR, mientras que en los hipertensos un $51 \%$ eran SS y $33 \%$ SR. Este mismo grupo ha comprobado la reproducibilidad del test, y la congruencia entre la respuesta a la repleción y depleción agudas con la obtenida por una repleción oral de $11 \mathrm{~g}$ de sal por 5 días seguida de 7 días de ingestas menores a $1 \mathrm{~g}$ Dentro de los SS Hollenberg y Williams describieron sujetos moduladores (M) y no moduladores (NM), según su respuesta renal y suprarrenal a una sobrecarga de sodio21. Los NM no ajustan su flujo renal con cambios de ingesta de sodio, y su respuesta de aldosterona a la reducción de sodio es reducida. Representan un subgrupo caracterizado por sensibilidad a la sal y respuesta al bloqueo del SRA. Tienen además mayor riesgo cardiovascular, microalbuminuria, resistencia a la insulina y aumento del contratransporte $\mathrm{Na}-\mathrm{Li}^{22}$. En ellos el uso de inhibidores de enzima de conversión restaura la capacidad del riñón de manejar la carga de sodio y reduce la presión arterial.

\section{Defectos del manejo tubular del sodio}

Durante la última década estudios de linkage ${ }^{2}$, o ligazón, y de positional cloning ${ }^{3}$, o posicionamiento clonal, han asociado alrededor de 20 genes a hipertensión arterial esencial o a formas de hipertensiones infrecuentes con herencia mendeliana ${ }^{23}$. La mayoría de estos genes codifican proteínas involucradas en el control renal de la reabsorción de $\mathrm{Na}$ en el túbulo renal (canales iónicos o componentes de sistemas endocrinos, o paracrinos, que intervienen en el manejo tubular del sodio). El polimorfismo G460W

\footnotetext{
${ }^{2}$ El análisis de linkage consiste en la búsqueda de genes que son heredados en conjunto, debido a su proximidad en un cromosoma específico. La enfermedad o genotipo se asocia, segrega o "viaja" con el alelo identificado.

${ }^{3}$ El positional cloning permite realizar un mapa de alta resolución para acotar la región y definir los genes responsables.
} 
del gen que codifica la unidad $\alpha$-aducina, que integra la bomba $\mathrm{Na} / \mathrm{K}$ del túbulo proximal, correlaciona con sensibilidad a la sal, actividad de renina baja, y una mayor respuesta a hidroclorotiazida.

Otro canal de $\mathrm{Na}$ susceptible de alteraciones génicas es el canal de $\mathrm{Na}$ sensible a amiloride (ENaC o Epithelial Na Channel), que controla la reabsorción de $\mathrm{Na}$ en el segmento conector y colector del túbulo renal. La mayor frecuencia del polimorfismo T594M de la subunidad $\beta$ en los hipertensos afro-americanos apoya una selección genética 24 . Las mutaciones de las subunidades $\beta$ y $\gamma$ se asocian al infrecuente Síndrome de Liddle, una hipertensión con renina y aldosterona suprimidas, respondedora a restricción de sal y a amiloride.

\section{Factores endocrinos y paracrinos que afectan la excreción de sodio}

Son varias las alteraciones de la síntesis de aldosterona que causan retención de $\mathrm{Na}^{25}$. La forma clínica más acentuada es el hiperaldosteronismo primario, causado por el adenoma suprarrenal, seguido por la hiperplasia nodular, la hiperplasia difusa, y el hiperaldosteronismo inapropiado para el nivel de renina. Existen defectos genéticos que determinan un aumento de la acción mineralocorticoidea, como el gen híbrido que otorga sensibilidad a ACTH a la aldosterona sintetasa, las alteraciones en la $11 \beta$ y la $17 \alpha$ hidroxilasa que elevan los niveles de deoxicorticosterona, y los defectos en la síntesis de cortisol por alteración de $11 \beta$-hidroxiesteroide dehidrogenasa que interfieren con el paso de cortisol a cortisona, favoreciendo su acumulación y su acción mineralocorticoidea.

El sistema calicreína-cininas, que ejerce un efecto natriurético, se expresa en el segmento conector de los túbulos distales 26 . Cepas deficientes en cininógeno y animales knock-out para el receptor 2 de bradicinina revelaron que el sistema calicreína-cininas recién induce natriuresis cuando se produce una marcada retención de sodio resultante de una elevada ingesta o de exceso de aldosterona. Al contrario, la estimulación de la síntesis tubular de calicreina por suplementación de potasio se acompaña de natriuresis 27,28 . Los hipertensos SS presentan una menor excreción de calicreina; los afro-americanos presentan mayor prevalencia de SS, menor ingesta de $\mathrm{K}$ y de calicreína urinaria y mayor reducción de presión con suplementación de $\mathrm{K}^{29}, 30$. La estimulación de calicreína renal por K está posiblemente involucrada en el mayor descenso tensional que se logra al disminuir la relación $\mathrm{Na} \mathrm{K}$, que el obtenido solamente al reducir el $\mathrm{Na}$.

\section{Otras condiciones que favorecen la reabsorción de sodio}

La excreción de Na también se modifica al disminuir el número de nefrones. Se ha constatado que los hipertensos tienen menor población nefronal. Además, sujetos que han nacido con bajo peso, que tienen más riesgo de desarrollar hipertensión en la vida adulta, también tendrían un menor número de nefrones ${ }^{31}$. Las glomerulopatías, obsolescencia glomerular del anciano, pérdidas de masa renal de otras causas (cirugía, reflujo) se acompañan de retención de Na y expansión de volumen.

Recientemente, se ha descrito una relación entreinflamación e hipertensión SS, la que se apoya en: 1) la asociación de citoquinas proinflamatorias como NF-kB y TNF- $\alpha$ e hipertensión de ratas Dahl sal sensibles, y 2) la immunosupresión mejora la hipertensión sal sensible y el aumento de Angiotensina II intrarrenal, probablemente debido a inflamación túbulo-intersticial32. Además, una ingesta elevada de sodio aumenta el stress oxidativo a través de la oxidasa $\mathrm{NAD}(\mathrm{P}) \mathrm{H}$, inhibiendo el transporte de L-arginina y a las sintasas de óxido nítrico.

La obesidad correlaciona positivamente con la reabsorción de sal en el túbulo proximal. Entre los mecanismos que explican esta mayor reabsorción están la activación del sistema renina-angiotensina y del sistema adrenérgico, la hiperinsulinemia y cambios morfológicos ${ }^{33}$ (fibrosis mesangial, compresión medular por aumento de la matriz intersticial).

El estudio CARDIAC mostró correlación entre ingesta y presión arterial para sistólica y diastólica en hombres, que en mujeres sólo aparece después de la menopausia. En mujeres que han experimentado una menopausia fisiológica o quirúrgica se ha observado que aumenta la sensibilidad a la sal, sugiriendo que los esteroides sexuales influencian la excreción de sodio y la presión arterial, y que su déficit promueve 
un desbalance entre óxido nítrico y angiotensina II que altera el manejo renal de sodio; esta mayor reabsorción revierte con la suplementación hormonal ${ }^{34,35}$.

La hipertensión geriátrica es habitualmente salsensible, con una alta frecuencia de hipertensión sistólica aislada36. A pesar de que la sensibilidad gustativa a la sal disminuye con la edad, la hipertensión no resulta de un aumento compensatorio en la ingesta, sino de una inhabilidad para excretar $\mathrm{Na}$, por disminución de función renal y de la actividad de la Na-K-adenosina trifosfatasa (Na/K-ATPasa), con aumento de $\mathrm{Na}$ y $\mathrm{Ca}$ intracelular..

\section{Factores que modifican el contenido de sodio intracelular}

Los niveles de ouabaina endógena, hormona de la corteza suprarrenal que inhibe selectivamente la bomba de $\mathrm{Na}$, están elevados en, aproximadamente, el $40 \%$ de los hipertensos 19 . Agentes que interfieren con la acción de ouabaina sobre la bomba $\alpha 2$ de $\mathrm{Na}$, o que reducen la expresión de la bomba $\mathrm{Na} / \mathrm{Ca}$ impiden el desarrollo de hipertensión sal sensible o de aquella dependiente de ouabaina, y son potencialmente atractivos para manejar la hipertensión.

\section{Acción del sodio en el sistema nervioso central}

El Na no sólo contribuye a expandir el volumen plasmático y la reactividad vascular, sino que también aumenta el tono del centro vasomotor. Ferrario y su grupo infundieron soluciones hipertónicas en el tercer ventrículo de ratas Sprague Dawley por 7 días, y demostraron un incremento de presión sistólica de $23 \pm 5 \mathrm{~mm} \mathrm{Hg}$ en el $1^{\text {er }}$ día y $15 \pm 2 \mathrm{~mm} \mathrm{Hg}$ en el $7^{\mathrm{mo}}$, además de mayor ingesta hídrica, hiponatremia, descenso de osmolaridad plasmática y elevación de norepinefrina plasmática 37,38 . La respuesta a la inyección intraventricular de $\mathrm{Na}$ difiere si se inyecta en el tercer ventrículo o la cisterna magna. En ambos casos se eleva la presión arterial y la frecuencia cardíaca, pero si la inyección se efectúa en la cisterna magna no se altera la estimulación simpática renal ni las secreciones hipofisiarias. Además, los barorreceptores responden a cambios en el balance de $\mathrm{Na}$, exacerbando su capacidad inhibitoria con la disminución de la ingesta, y reduciéndola con alta ingesta 39 .

\section{Efectos no presores de la sal}

La alta ingesta de sal se asocia a mayor masa ventricular izquierda, tanto en normotensos como en hipertensos, independientemente de la presión arterial 40 . Los mecanismos que generan el aumento de la masa ventricular son aún desconocidos, pero estarían relacionados con un aumento de la sensibilidad de los receptores $\alpha$ adrenérgicos del intercambiador $\mathrm{Na} / \mathrm{H}$. También se ha observado que la ingesta de sodio se correlaciona con la excreción urinaria de albúmina, indicador de riesgo cardiovascular. Además, la alta ingesta de sal se ha asociado a una mayor prevalencia de cáncer gástrico, y su reducción integra las medidas preventivas para esta prevalente neoplasia41, 42. Probablemente, este efecto se debe a la selección de una cepa de Helycobacyer Pylori más oncogénica, la que expresa una proteína, CagA, y un sistema de secreción que la entrega a las células del huésped, alterando su morfología, la proliferación y la vía mitogénica de la kinasa extracelular (ERK); así CagA funciona como una oncoproteína bacteriana ${ }^{43}$.

\section{Recomendaciones}

Se debate si la restricción de la ingesta de sal a toda la población lograría efectos beneficiosos, pues incluiría a sujetos insensibles a la sal. Además, se postula que una reducción excesiva del aporte de sal puede ser deletérea ${ }^{44}$. Si bien una ingesta diaria de $1 \mathrm{~g}$ se acompaña de elevación de colesterol total y LDL, hemoglobina, proteínas totales, albúmina, norepinefrina, insulina y relación glucosa/insulina, la cantidad recomendada entre 4 a 6 g 45,46 está lejos de provocar estimulación adrenérgica. Una estrategia para reforzar la reducción de la ingesta de sal en hipertensos y en normotensos con presiones normales altas, es recomendar que esta reducción a toda la familia. Después de conocer los distintos genotipos responsables de la retención de sal, debemos considerar al individuo hipertenso como un caso índice de una tendencia familiar, y estamos obligados a instalar medidas preventivas en sus hijos.

Si bien aún no existe una intervención simple para determinar la sensibilidad o insensibilidad a la sal, 


\section{G.Valdés}

tenemos considerar que la ingesta de sal en Chile es elevada, debido a la importante ingesta de pan y al hábito de salar generosamente la comida antes de probarla. Diversos estudios han corroborado excreciones urinarias de aproximadamente $10 \mathrm{~g} \mathrm{Na}$. Esta cifra sobrepasa los $9 \mathrm{mmol}$ diarios definidos como la cantidad que contribuye a refractariedad a tratamiento antihipertensivo, y es perentorio disminuirla.

\section{Conclusión}

Es claro que la sal, y el manejo que de este ión hace el riñón, constituyen la causa más importante de elevación de las cifras tensionales. Existiendo tantas vías para retener $\mathrm{Na}$, lo más lógico es pensar que la sensibilidad a la sal está determinada por distintos genotipos, actuando en forma monogénica en pequeños grupos de hipertensos, y en combinaciones poligénicas que interactúan en la mayoría con el fenotipo (sexo, edad, obesidad, reducción de función renal). Estos genotipos se habrían acumulado a lo largo de la evolución para permitir la sobrevida en condiciones de bajo aporte, pero en la actualidad otorgan un importante riesgo para desarrollar hipertensión. Aún cuando hasta el momento no podamos identificar a los individuos sal-sensibles con intervenciones sencillas, el beneficio comprobado de una restricción moderada y los efectos no presores de la sal, justifican ampliamente la recomendación de una ingesta entre 3.5 y $6 \mathrm{~g}$ diarios.

\section{Referencias}

1. ALDERMAN M. A pinch of science. Artículo de opinión. New York Times, 6 Febrero 2009.

2. STAMLER J, ROSE G, ELLIOTT P, DYER A, MARMOT M, KESTELOOT $H$, et al. Findings of the international cooperative intersalt study. Hypertension. 1991; 17: 19-15.

3. STAMLER J. The Intersalt study: Background, methods, findings, and implications. Am J Clin Nutr. 1997; 65: 626S-642S.

4. YAMORI Y, NARA Y, MIZUSHIMA S, MANO M, SAWAMURA M, KIHARA M, et al. International cooperative study on the relationship between dietary factors and blood pressure: A report from the cardiovascular diseases and alimentary comparison (CARDIAC) study

J Cardiovasc Pharmacol. 1990; 16 Suppl 8: S43-47.

5. YAMORI Y, LIU L, IKEDA K, MIZUSHIMA S, NARA Y, SIMPSON FO. Different associations of blood pressure with 24-hour urinary sodium excretion among pre- and post-menopausal women. WHO cardiovascular diseases and alimentary comparison (WHO-CARDIAC) study. J Hypertens. 2001; 19: 535-538.

6. POULTER NR, KHAW KT, MUGAMBI M, PEART WS, SEVER PS. Migration-induced changes in blood pressure: A controlled longitudinal study. Clin Exp Pharmacol Physiol. 1985; 12: 211-216.

7. HE J, TELL GS, TANG YC, MO PS, HE GQ. Relation of electrolytes to blood pressure in men. The yi people study. Hypertension. 1991; 17: 378-385.

8. FORTE JG, MIGUEL JM, MIGUEL MJ, DE PADUA F, ROSE G. Salt and blood pressure: A community trial. J Hum Hypertens. 1989; 3: 179-184.

9. WILSON TW, GRIM CE. Biohistory of slavery and blood pressure differences in blacks today. A hypothesis. Hypertension. 1991; 17: I122-128.

10. KEMPNER W. Treatment of hypertensive vascular disease with rice diet. Arch Intern Med. 1974; 133: 758-790.

11. HE FJ, MACGREGOR GA. Effect of modest salt reduction on blood pressure: A meta-analysis of randomized trials. Implications for public health.

J Hum Hypertens. 2002; 16: 761-770.

12. AKITA S, SACKS FM, SVETKEY LP, CONLIN PR, KIMURA G. Effects of the dietary approaches to stop hypertension (DASH) diet on the pressure-natriuresis relationship. Hypertension. 2003; 42: 8-13.

13. GUYTON AC, MANNING RD, HALL JE, NORMAN RA, YOUNG DB, PAN YJ. The pathogenic role of the kidney. J Cardiovasc Pharmacol. 1984; 6: S151-161.

14. GUYTON AC. Dominant role of the kidneys and accessory role of whole-body autoregulation in the pathogenesis of hypertension. Am J Hypertens. 1989; 2: 575-585.

15. GUYTON AC, COLEMAN TG. Quantitative analysis of the pathophysiology of hypertension. 1969.

J Am Soc Nephrol. 1999; 10: 2248-2258.

16. GUIDI E, COZZI MG, MINETTI E, BIANCHI G. Donor and recipient family histories of hypertension influence renal impairment and blood pressure during acute rejections. J Am Soc Nephrol. 1998; 9: 2102-2107.

17. TOBIAN L. The electrolytes of arterial wall in experimental renal hypertension. Circ Res. 1956; 4: 671-675.

18. BLAUSTEIN MP. Sodium ions, calcium ions, blood pressure regulation, and hypertension: $A$ reassessment and a hypothesis. Am J Physiol. 1977; 232: C165-173. 
19. BLAUSTEIN MP, ZHANG J, CHEN L, SONG H, RAINA H, KINSEY SP, et al. The pump, the exchanger, and endogenous ouabain: Signaling mechanisms that link salt retention to hypertension. Hypertension. 2009; 53: 291-298.

20. LUFT FC, WEINBERGER MH. Heterogeneous responses to changes in dietary salt intake: The salt-sensitivity paradigm. Am J Clin Nutr. 1997; 65: 612S-617S.

21. WILLIAMS GH, HOLLENBERG NK. Sodium-sensitive essential hypertension: Emerging insights into an old entity. J Am Coll Nutr. 1989; 8: 490-494.

22. SANCHEZ RA, GIANNONE C, MASNATTA LD, BAGLIVO HP, RAMIREZ AJ. Higher urinary albumin excretion is associated with abnormal erythrocyte na(+)/li(+) countertransport (slc) in non-modulating essential hypertensives and offspring of hypertensive parents. J Hum Hypertens. 2002; 16: S128-132.

23. MENETON P, JEUNEMAITRE X, DE WARDENER HE, MACGREGOR GA. Links between dietary salt intake, renal salt handling, blood pressure, and cardiovascular diseases. Physiol Rev. 2005; 85: 679-715.

24. BAKER EH, DONG YB, SAGNELLA GA, ROTHWELL M, ONIPINLA AK, MARKANDU ND, et al. Association of hypertension with $\mathrm{t} 594 \mathrm{~m}$ mutation in beta subunit of epithelial sodium channels in black people resident in london. Lancet. 1998; 351: 1388-1392.

25. FARDELLA CE, MOSSO L, GOMEZ-SANCHEZ C, CORTES P, SOTO J, GOMEZ L, et al. Primary hyperaldosteronism in essential hypertensives: Prevalence, biochemical profile, and molecular biology.

J Clin Endocrinol Metab. 2000; 85: 1863-1867.

26. FIGUEROA CD, CAORSI I, SUBIABRE J, VIO CP. Immunoreactive kallikrein localization in the rat kidney: An immuno-electron-microscopic study. J Histochem Cytochem. 1984; 32: 117-121.

27. VALDES G, VIO CP, MONTERO J, AVENDANO R. Potassium supplementation lowers blood pressure and increases urinary kallikrein in essential hypertensives.

J Hum Hypertens. 1991; 5: 91-96.

28. ARDILES LG, FIGUEROA CD, MEZZANO SA. Renal kallikrein-kinin system damage and salt sensitivity: Insights from experimental models. Kidney Int Suppl. 2003; 86: S2-8.

29. SONG CK, MARTINEZ JA, KAILASAM MT, DAO TT, WONG CM, PARMER RJ, et al. Renal kallikrein excretion: Role of ethnicity, gender, environment, and genetic risk of hypertension. J Hum Hypertens. 2000; 14: 461-468.

30. BRANCATI FL, APPEL LJ, SEIDLER AJ, WHELTON PK. Effect of potassium supplementation on blood pressure in african americans on a low-potassium diet. A randomized, double blind, placebo-controlled trial. Arch Intern Med. 1996; 156: 61-67.

31. MANALICH R, REYES L, HERRERA M, MELENDI C,
FUNDORA I. Relationship between weight at birth and the number and size of renal glomeruli in humans: $A$ histomorphometric study. Kidney Int. 2000; 58: 770-773.

32. FRANCO M, SANCHEZ-LOZADA LG, BAUTISTA R, JOHNSON RJ, RODRIGUEZ-ITURBE B. Pathophysiology of salt-sensitive hypertension: A new scope of an old problem. Blood Purif. 2008; 26: 45-48.

33. WOFFORD MR, HALL JE. Pathophysiology and treatment of obesity hypertension.

Curr Pharm Des. 2004; 10: 3621-3637.

34. SCHULMAN IH, ARANDA P, RAIJ L, VERONESI M, ARANDA FJ, MARTIN R. Surgical menopause increases salt sensitivity of blood pressure.

Hypertension. 2006; 47: 1168-1174.

35. SCUTERI A, LAKATTA EG, ANDERSON DE, FLEG JL. Transdermal 17 beta-oestradiol reduces salt sensitivity of blood pressure in postmenopausal women.

J Hypertens. 2003; 21: 2419-2420.

36. ZEMEL MB, SOWERS JR. Salt sensitivity and systemic hypertension in the elderly. Am J Cardiol. 1988; 61: $7 \mathrm{H}-12 \mathrm{H}$.

37. BRUM JM, TRAMPOSCH AF, FERRARIO CM. Neurovascular mechanisms and sodium balance in the pathogenesis of hypertension. Hypertension. 1991; 17: 145-51.

38. KAWANO Y, SUDO RT, FERRARIO CM. Effects of chronic intraventricular sodium on blood pressure and fluid balance. Hypertension. 1991; 17: 28-35.

39. FERRARIO CM, TRAMPOSCH A, KAWANO Y, BROSNIHAN $\mathrm{KB}$. Sodium balance and the reflex regulation of baroreceptor function. Circulation. 1987; 75: I141-148.

40. HARMSEN E, LEENEN FH. Dietary sodium induced cardiac hypertrophy. Can J Physiol Pharmacol. 1992; 70: 580-586.

41. SHIKATA K, KIYOHARA Y, KUBO M, YONEMOTO K, NINOMIYA T, SHIROTA T, et al. A prospective study of dietary salt intake and gastric cancer incidence in a defined japanese population: The hisayama study. Int J Cancer. 2006; 119: 196-201.

42. TSUGANE S, SASAZUKI S, KOBAYASHI M, SASAKI S. Salt and salted food intake and subsequent risk of gastric cancer among middle-aged japanese men and women. Br J Cancer. 2004; 90: 128-134.

43. KATOH M. Dysregulation of stem cell signaling network due to germline mutation, snp, helicobacter pylori infection, epigenetic change and genetic alteration in gastric cancer Cancer Biol Ther. 2007; 6: 832-839.

44. ALDERMAN MH. Dietary sodium and cardiovascular health in hypertensive patients: The case against universal sodium restriction. J Am Soc Nephrol. 2004; 15: S47-50

45. APPEL LJ, BRANDS MW, DANIELS SR, KARANJA N, ELMER PJ, SACKS FM. Dietary approaches to prevent and treat hypertension: A scientific statement from the american heart association. Hypertension. 2006; 47: 296-308. 
G.Valdés

46. KAWANO Y, ANDO K, MATSUURA H, TSUCHIHASHI T, FUJITA T, UESHIMA H. Report of the working group for dietary salt reduction of the japanese society of hypertension: (1) rationale for salt restriction and saltrestriction target level for the management of hypertension. Hypertens Res. 2007;30: 879-886. 